\title{
South American SLR stations monitoring ground displacement caused by the M8.8 Chilean earthquake of 2010
}

\author{
YIN ZhiQiang $^{1 *}$, HAN YanBen $^{1 *}$, PODESTÁ Ricardo ${ }^{2}$, LIU WeiDong ${ }^{1}$, PACHECO Ana ${ }^{2}$, \\ ALONSO Ester ${ }^{2}$, ACTIS Eloy ${ }^{2}$, HUANG DongPing ${ }^{1}$, ZHAO LiMin $^{1}$, WANG Rui ${ }^{1} \&$ \\ XIA JinChao ${ }^{1}$ \\ ${ }^{1}$ National Astronomical Observatories, Chinese Academy of Sciences, Beijing 100012, China; \\ ${ }^{2}$ Observatorio Astronomico Felix Aguilar, San Juan University, San Juan 5400, Argentina
}

Received September 21, 2010; accepted November 14, 2010

\begin{abstract}
The M8.8 Chilean earthquake of February 27, 2010 caused a large surface displacement near its epicenter. Satellite Laser Ranging (SLR) station 7405, cooperatively operated by Germany and Chile about $130 \mathrm{~km}$ from the epicenter was affected by the earthquake. Another SLR station, No. 7406, operated jointly by the National Astronomical Observatories of the Chinese Academy of Sciences and San Juan University of Argentina, was about $600 \mathrm{~km}$ from the epicenter. Combining the observations of these two SLR stations with global SLR station observations, we calculate the geocentric coordinates of the two stations based on ITRF2000. The results show that the coordinates of the two stations have changed by varying degrees. SLR-7405 moved substantially towards the southwest with displacements of about 3.11, 0.52 and $0.49 \mathrm{~m}$ in $X, Y, Z$ directions; the corresponding displacements for SLR-7406 are about $0.02,0.03$ and $0.02 \mathrm{~m}$. This solution, as an independent result derived from SLR observations, could provide an essential external check for other positioning techniques such as GPS.
\end{abstract}

earthquake, satellite laser ranging (SLR), ITRF2000, geocentric coordinates, ground displacement in South America

Citation: $\quad$ Yin Z Q, Han Y B, Podestá R, et al. South American SLR stations monitoring ground displacement caused by the M8.8 Chilean earthquake of 2010. Chinese Sci Bull, 2011, 56: 738-742, doi: 10.1007/s11434-010-4324-8

The M8.8 Chilean earthquake of February 27, 2010 not only caused major damage near the epicenter, triggering a tsunami that devastated several coastal towns in south-central Chile, but also produced obvious ground displacement near to the epicenter. According to related reports, GPS measurements showed that the entire city of Concepción moved as much as $3 \mathrm{~m}$ to the west. The country's capital, Santiago, moved about $30 \mathrm{~cm}$ to the west. Even Buenos Aires, nearly $1300 \mathrm{~km}$ from the epicenter, and some other areas of Argentina also shifted measurably to the west. This earthquake area is of particular interest to geoscientists because it lies above an active subduction zone, where the Nazca Plate is colliding with the South American tectonic plate and is pushed down into the Earth's mantle below. Because of the

*Corresponding authors (email: yinzhq@nao.cas.cn; hyb@nao.cas.cn) recent earthquake, this region of seismic activity has become a focus of attention for Earth scientists.

Precise GPS measurements of crustal movement at plate boundaries are becoming routine in many tectonically active regions. Other new space measuring technologies, such as very long baseline interferometry (VLBI) and satellite laser ranging (SLR) can also give high-precision observations. Of these, SLR has the most accurate measurement in singlepoint sampling, in modern measuring and position technologies. SLR observations are important in the detection of crustal movement, Earth's gravity field variation, the precise International Terrestrial Reference Frame (ITRF) maintenance and other aspects [1-6].

For the Chilean earthquake, the International Laser Ranging Service (ILRS) SLR station 7405, cooperatively operated by Germany and Chile, is coincidently located in 
Concepción, near the epicenter. About $700 \mathrm{~km}$ northeast of station 7405, SLR observatory 7406 is operated by China and Argentina under another cooperative agreement between the National Astronomical Observatories of Chinese Academy of Sciences (NAOC) and the San Juan University (Figure 1). The main telescope aperture of SLR-7405 is 50 $\mathrm{cm}$; this equipment is in good operating condition and has made abundant observations since its commissioning in May 2002. Data support the position of station SLR-7405 among the top ten SLR systems in the global ILRS network. SLR-7406, with a 60-cm receiving telescope, was constructed by China and installed at the San Juan Observatory in Argentina at the end of 2005. The San Juan Observatory built the observational room. The SLR started to observe at the end of February 2006. Two or three NAOC scientists routinely are present at the observatory where they are responsible for equipment maintenance and observation. Superior observational results in San Juan have been due to the excellence of the observational equipment and personnel, as well as optimal observational weather in the San Juan area of Argentina. Data support the position of station SLR-7406 among the top five SLR systems in the global ILRS network [7].

In this paper, we utilize the data from the satellite Lageos-1 observed by the two SLRs before and after the earthquake to calculate the variation of the geocentric coordinates of the two SLRs, and to obtain their ground displacements due to the earthquake.

\section{Observational data}

The geodetic coordinates of SLR-7405 are $\varphi=-36.843^{\circ}, \lambda$ $=-73.025^{\circ}$, about $130 \mathrm{~km}$ away from the epicenter $(\varphi=$ $-35.7^{\circ}, \lambda=-72.7^{\circ}$ ) of the $M 8.8$ Chilean earthquake. This SLR was struck hard by the major earthquake, but was fully operational again just over two months later, at the beginning of May 2010. The geodetic coordinates of SLR-7406

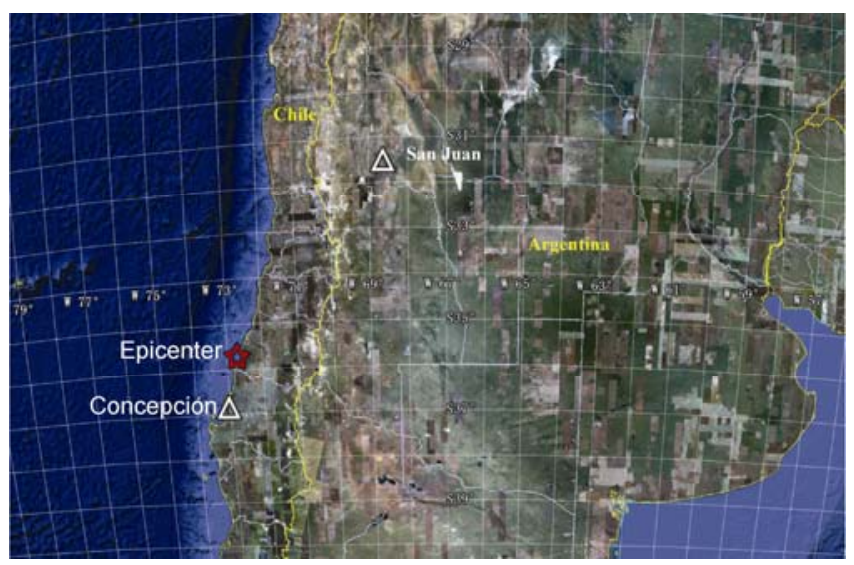

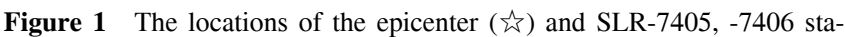
tions $(\triangle)$. are $\varphi=-31.509^{\circ}, \lambda=-68.623^{\circ}$, about $600 \mathrm{~km}$ away from the epicenter. The observations at this station were not interrupted by the earthquake. The distance between SLR-7405 and SLR-7406 is about $716 \mathrm{~km}$.

The Lageos-1 laser-ranging satellite was designed especially for geodynamic research and is also one of the preferred satellites for the global SLR network. Therefore, observations from of Lageos- 1 are abundant as well as highly precise. To compare the variation of SLR station movement before and after the earthquake, we selected Lageos-1 data supported by all the SLR stations during the period from 2009-01-01 to 2010-06-22 (http://ilrs.gsfc.nasa.gov/), and the observations of SLR-7405 are the approximate twomonth-long period after the earthquake.

\section{Determination of precise orbit and station coordinates}

Methods of accurately determining the orbit of Lageos- 1 are undertaken based on similar sets of input parameters [8,9]. The reference frame, measurement and force models basically follow the IERS conventions.

(i) Force models. In the development of force models, the following perturbation factors are considered: (1) lunar and solar gravity perturbations, (2) solid Earth tide, (3) ocean tide (CSR4.0), (4) the GGM02C Earth gravity field model, (5) solar and Earth radiation pressure $(\mathrm{Cr}$ and derivative $\mathrm{Cr}$ adjusted), (6) the drag-like perturbation ( $\mathrm{Cd}$ and derivative $\mathrm{Cd}$ adjusted), (7) thermal radiation imbalances from the Earth and sun, (8) general relativistic perturbation, and (9) Earth's rotation deformation perturbation. To improve the precision of the orbital determination, the empirical perturbations due to periodic empirical radial and transverse accelerations are also considered.

(ii) Measurement models. Measurement models are considered as follows: (1) the Marini-Murray refraction model, (2) the offset correction about the center of mass of Lageos-1, $0.251 \mathrm{~m}$, (3) station displacement from solid Earth tides, (4) the influence of ocean loading at each site, (5) the influence of permanent tide deformation at each site, and (6) rotational deformation due to polar motion.

(iii) Reference frame. The reference frame utilizes (1) the mean equinox ( $X$-direction) and equator $(X-Y$ plane) of J2000.0, (2) the precession constants of IAU 1976, (3) the nutation coefficients from the IAU 1980 theory of nutation and the celestial pole offset of IERS, (4) the DE403/LE403 planetary ephemeris, and (5) the initial values of station coordinates from ITRF2000.

(iv) Multistage multi-arc method. To improve the precision and efficiency of the orbital determination, the multistage multi-arc method is adopted for the data calculation. The parameters to be estimated are (a) a set of orbital elements of Lageos-1 and coordinates of 7405 and 7406 stations per 15-day arc, (b) solar and terrestrial radiation coefficients 
and their rates per 15-day arc, (c) Earth orientation parameters (EOP) per 3-day arc, and (d) the coefficients of empirical RTN and drag-like coefficient per 1-day arc.

\section{Analysis of results}

As the record for station 7405 near the epicenter extends for only about two months after the earthquake, more independent solutions are needed. Considering the stability of the SLR observational data, we selected a period of 15 days as a unified arc through many test to process the precise orbital determination and calculate coordinates. The time span of the data is from January 1, 2009 to June 22, 2010. Generally, Australia's SLR-7090 station has the highest quality in the ILRS network stations and is weighted more highly in the data processing. However, on December 7, 8, 10 and 11, 2009 the station experienced larger deviations in the data, so these four days were deleted to avoid adversely influencing the result. In the calculation, only the coordinates of stations 7405 and 7406 are estimated, whereas the coordinates of other SLR stations are regarded as fixed. We then get 35 independent results; the quantity of each and the statistics of the orbit residual RMS values are plotted in Figures 2 and 3, respectively. The total number of the data

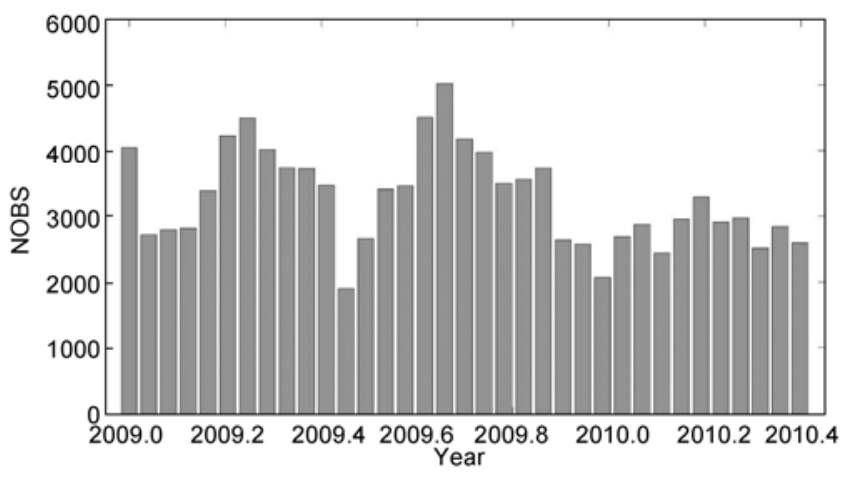

Figure 2 Statistics of the number of Lageos-1 data per 15-day arc (time span 2009-01-01-2010-06-22).

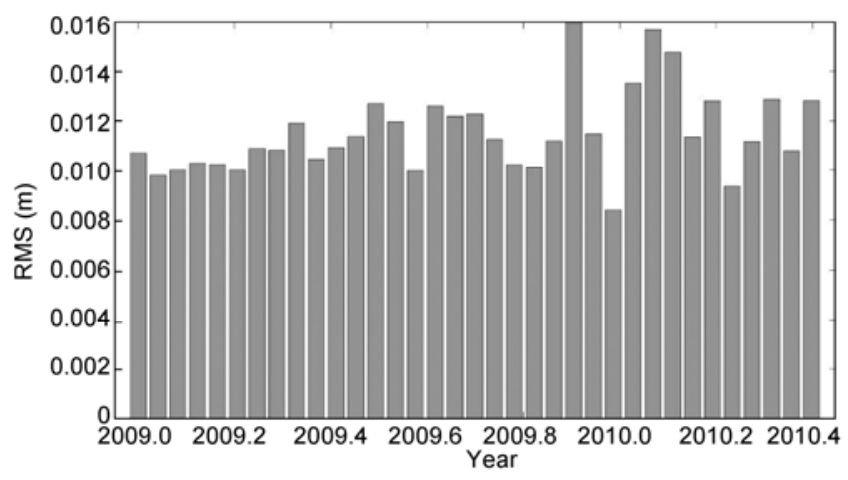

Figure 3 Statistics of orbit residual RMS values of Lageos-1 (time span 2009-01-01-2010-06-22).
(NOBS) is 114746 , the average of the NOBS is 3278 , and the mean of residual RMS is $0.012 \mathrm{~m}$.

As the satellite observational data obtained by the global SLR stations are not uniform in time, observations of the Lageos-1 satellite are further processed over 1-day intervals. The scheme used for the determination of precise orbit and station coordinates is the same; the arc is still 15-days long. The output is the time series of the coordinates of stations 7405 and 7406 based on ITRF2000 (Figure 4). Several obviously biased solutions caused by too few observations or the low precision in the original data were deleted.

Figure 4 shows that SLR-7405 in Concepción, near the epicenter, was measurably moved due to the $M 8.8$ Chilean earthquake in 2010. The displacement is more than $3 \mathrm{~m}$ in the $X$ direction, and is about $0.5 \mathrm{~m}$ in the $Y$ and $Z$ directions. San Juan station 7406, separated from station 7405 by about $700 \mathrm{~km}$, also moved. The displacements are relatively obvious in both $X$ and $Y$ directions. The statistical results of the above calculations are shown in Table 1.

Table 1 lists the averages of the results, standard deviations (STD), and the difference in stations coordinates before and after the earthquake. STD of $\Delta X, \Delta Y$ and $\Delta Z$ mainly represent the accuracy of the internal agreement of $X$, $Y$ and $Z$, respectively. They basically show the precision of the fit between the solutions of the coordinates of each site and the satellite's orbits and the observational data; the STD is about $2 \mathrm{~cm}$. SLR-7405 and SLR-7406 began to operate in 2002 and 2006, respectively. As new sites, they joined the updated ITRF2005 and ITRF2008 reference frames (http://itrf.ensg.ign.fr/). To compare the present results with the recent reference frame, we selected the two stations' coordinates in ITRF2008 and calculated their values in ITRF2000, at epoch 1997 through a Bursa seven-parameter transformation. The results are presented in the "ITRF08to2k" column of Table 1. This provides an external test of precision, and shows that the difference between ITRF2000 and the present results are about $2 \mathrm{~cm}$.

\section{Conclusions and discussion}

The subduction zone between the Nazca and South American plates belongs to the circum-Pacific seismic belt. Major earthquakes occur there frequently, and the area is the focus of much global research. SRL-7405 and SRL-7406 are outstanding SLR stations within the ILRS network. After analysis of their observations before and after the M8.8 Chilean earthquake of 2010, station SLR-7405 was determined to have moved $3.11,0.52$ and $0.49 \mathrm{~m}$ in $X / Y / Z$ directions, while station SLR-7406 moved about 0.02, 0.03 and $0.02 \mathrm{~m}$. These results show that the movement of the local crustal was linked with the SLR results before and after the earthquake. The displacement near the epicenter was larger; stations farther from the epicenter were less affected. Solutions derived from GPS measurements indicate that the 

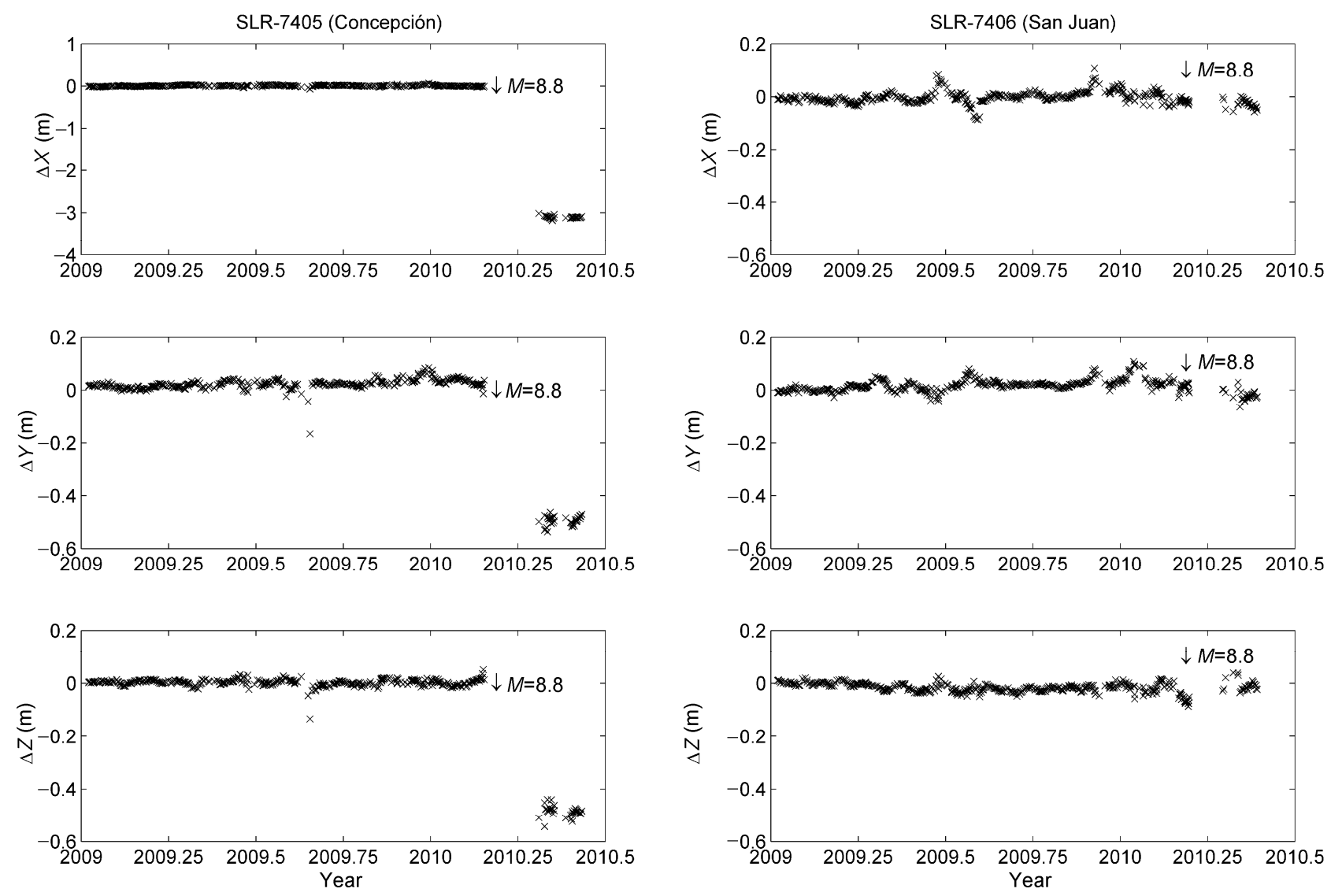

Figure 4 The variations of geocentric coordinates of Concepción and San Juan before and after the $M 8.8$ Chilean earthquake in 2010. Geocentric coordinates are represented in an $X, Y$ and $Z$ coordinate frame. The center of mass of the Earth is the coordinates' origin. The $Z$-axis is parallel to the Earth's rotational axis and points north. The $X$-axis points towards the point of intersection between the Greenwich meridian plane and the equator of the Earth, and the $X / Y I Z$ follows the right-handed orientation. $\Delta X, \Delta Y$ and $\Delta Z$ represent the accuracy of internal agreement of $X, Y$ and $Z$, respectively. The arrows indicate the earthquake moment.

Table 1 Variation of coordinates of SLR-7405 and SLR-7406 in ITRF 2000 before and after the M8.8 earthquake

\begin{tabular}{ccccccc}
\hline & $X(\mathrm{~m})$ & $Y(\mathrm{~m})$ & $Z(\mathrm{~m})$ & $\mathrm{STD}_{\Delta X}(\mathrm{~m})$ & $\mathrm{STD}_{\Delta Y}(\mathrm{~m})$ & $\mathrm{STD}_{\Delta Z}(\mathrm{~m})$ \\
\hline 7405 bef. EQ & 1492032.7583 & -4887946.0478 & -3803566.0389 & 0.014 & 0.018 & 0.012 \\
7405 aft. EQ & 1492029.6433 & -4887946.5663 & -3803566.5262 & 0.031 & 0.017 & 0.020 \\
7405 aft.-bef. & -3.114 & -0.5185 & -0.4873 & - & - & - \\
ITRF08to2k(7405) & 1492032.7512 & -4887946.0714 & -3803566.0414 & - & - & - \\
7405 bef. EQ-ITRF & 0.0071 & 0.0236 & 0.0025 & - & - & - \\
7406 bef. EQ & 1984104.2205 & -5068867.1380 & -3314482.6836 & 0.022 & 0.022 & 0.015 \\
7406 aft. EQ & 1984104.1988 & -5068867.1653 & -3314482.6986 & 0.014 & 0.023 & 0.031 \\
7406 aft.-bef. & -0.0217 & -0.0273 & -0.015 & - & - & - \\
ITRF08to2k(7406) & 1984104.2214 & -5068867.1582 & -3314482.6687 & - & - & - \\
7406 bef. EQ-ITRF & -0.0009 & 0.0202 & -0.0149 & - & - & - \\
\hline
\end{tabular}

a) bef. EQ means before the Chilean earthquake of 2010-02-27; aft. EQ means after the earthquake.

maximum movement near Concepción is $3.04 \mathrm{~m}$ to the southwest, similar to our calculation. There is no GPS data for the San Juan station, but at Mendoza $\left(\varphi=-32.89^{\circ}, \lambda=\right.$ $-68.84^{\circ}$ ), which lies closer to the epicenter to the south of San Juan station, the GPS station was determined to have moved about $13 \mathrm{~cm}$. Another GPS station near San Juan moved
$5.5 \mathrm{~cm}$ (http://www.soest.hawaii.edu/soest_web/soest.news_ chile_feb2010_eq.htm). The displacement of SLR-7406 is of the order of about $2 \mathrm{~cm}$, which also can compare with the GPS solutions. In fact, the results obtained by SLR act as independent measurements that can be compared with GPS and other positioning techniques to provide an external 
check of the other technique. In addition, the work presented here suggests that the velocities of the two SLR stations seem to have changed (Figure 4). However, this still needs further analysis because the time series of valid post-seismic position data do not extend over a sufficiently long observation period.

This earthquake took place along the boundary between the Nazca and South American tectonic plates, which are converging at a rate of $\sim 8 \mathrm{~cm}$ per year. This earthquake was characterized by a thrust-faulting focal mechanism, caused by the subduction of the Nazca plate beneath the South American [10,11]. It is noteworthy that the velocity of SLR-7405 station is $0.0319,-0.0014,0.0171 \mathrm{~m} / \mathrm{yr}$ in $X / Y / Z$ directions in ITRF2005, and 0.0349, $-0.0015,0.0169 \mathrm{~m} / \mathrm{yr}$ in ITRF2008. The data used to determine ITRF2005 are up to the end of 2005, and the data used for ITRF2008 reach the end of 2008. Both of them show that the velocity of SLR-7405 is maximized in the $X$ direction, which likely reflects the characteristic movements of the South American plate in this region. The subduction of the Nazca plate beneath the South American caused the earthquake and analysis of SLR observations shows there is the maximum displacement of nearly $3 \mathrm{~m}$ in the $X$ direction on the South American Plate after the earthquake.

The primary seismically active areas of Chile surround the subduction zone between the Nazca and South American tectonic plates. The historical record since AD 1570 documents 120 earthquakes with $M \geqslant 7.0$ in the region. Some studies of historic earthquakes indicate that the present day may mark the beginning of a seismically active period in this area, and another major earthquake over M8.0 might occur in the region during the next 3 or 4 years [10]. Future studies will benefit from the collaborative German-Chilean station 7405 that has SLR, VLBI, GPS and other techniques for co-location observations. Observations obtained by the variety of measurements made here more valuable for related scientific research. The NAOC and San Juan University collaboration with station 7406 will soon have a permanent GPS station near the SLR system that will enable co-locations from SLR and GPS observations. It also will use SLR and GPS observations to carry out related research $[7,12,13]$.

This work was supported by the Foundation of International Science and Technology Cooperation (2009DFB00130), the External Cooperation Program of the Chinese Academy of Sciences (GJHZ200813) and the Young Researcher Grant of NAOC.

1 Dong D N, Yunck T, Heflin M. Origin of the international terrestrial reference frame. J Geophys Res, 2003, 108: 2200-2209

2 Qin X P, Yang Y X, Wang G, et al. GLONASS orbit determination by using SLR data (in Chinese). Geomat Inform Sci Wuhan Univ, 2003, 28: 440-443

3 Zhu Y L, Feng C G. Earth orientation parameter and the geocentric variance during 1993-2002 solved with Lageos SLR data (in Chinese). Acta Geod Cartogr Sin, 2005, 34: 19-23

4 Feissel-Vernier M, Le Bail K, Berio P, et al. Geocentre motion measured with DORIS and SLR, and predicted by geophysical models. J Geodesy, 2006, 80: 637-648

5 Coulot D, Pollet A, Collilieux X. Global optimization of core station networks for space geodesy: Application to the referencing of the SLR EOP with respect to ITRF. J Geodesy, 2010, 48: 31-50

6 Guo J Y, Han Y B. Seasonal and inter-annual variations of length of day and polar motion observed by SLR in 1993-2006. Chinese Sci Bull, 2009, 54: 46-52

7 Han Y B, Liu W D, Actis E, et al. Successful operation of a cooperative SLR station of China and Argentina in San Juan. Chinese Sci Bull, 2008, 53: 2417-2420

8 Feng C G, Cai W S, Zhu Y L, et al. Determination of Chinese mobile SLR sites' coordinates. Chinese Sci Bull, 2003, 48: 2300-2302

9 Schillak S, Wnuk E. The SLR stations coordinates determined from monthly arcs of LAGEOS-1 and LAGEOS-2 laser ranging in 19992001. Adv Space Res, 2003, 31: 1935-1940

10 Ren J J, Zhou N. The 2010 (M8.8) Chile earthquake, the historic earthquakes and the tectonic setting (in Chinese). Recent Develop World Seism, 2010, 375: 1-7

11 Zhang Y, Xu L S, Chen Y T. Fast inversion of the rupture process of 27 February 2010 Chile earthquake (in Chinese). Acta Seism Sin, 2010, 32: 242-244

12 Liu W D, Han Y B, Podestá R, et al. The present and future of an SLR system built under the cooperation between China and Argentina (in Chinese). Astron Res Tech, 2009, 6: 1-7

13 Liu W D, Han Y B, Podestá R, et al. Actuality and futuruty of San Juan 7406 station cooperation between China and Argentina. In: Schillak S, ed. Proceedings of the 16th International Workshop on Laser Ranging, 2008 Oct 13-17, Poznań. Warsaw: Space Research Centre, Polish Academy of Sciences, 2009. 535-537 